\title{
INTERDEPENDENCIA ENTRE LA RESTAURACIÓN ECOLÓGICA Y LA CONSERVACIÓN NATURAL
}

\author{
INTERDEPENDENCE BETWEEN THE ECOLOGICAL RESTORATION AND \\ NATURE CONSERVATION
}

\section{INTERDEPENDANCE ENTRE LA RESTAURATION ECOLOGIQUE ET LA CONSERVATION NATURELLE}

\author{
Juan dos Santos \\ FLACSO Guatemala \\ jdossantos@flacso.edu.gt
}

(Tipo de artículo: REFLEXIÓN. Recibido el 12/03/2011. Aprobado el 30/05/2011)

\begin{abstract}
RESUMEN
La restauración ecológica se percibe por muchos conservacionistas, y por la mayoría de los economistas, como una diversión, una ilusión, y mucho peor, como una pérdida de tiempo y dinero. En este trabajo se señala que la restauración, de hecho, complementa no sólo a la conservación de la naturaleza sino que también es útil para lograr un desarrollo socio-económicamente sostenible y equitativo. Esto se debe a que restaurar y aumentar la base del capital natural es la mejor vía para generar puestos de trabajo, y mejorar los medios de supervivencia y de calidad de vida para todos, lo mismo que para la economía.
\end{abstract}

Palabras clave

Restauración ecológica, conservación natural, capital natural, conservación de las especies.

\section{ABSTRACT}

Ecological restoration is perceived by many conservationists, and by the majority of economists, as a diversion, an illusion, and much worse, as a loss of time and money. In this work we point out that the restoration, in fact, not only complements the conservation of nature but is also useful to achieve a socio-economically sustainable and equitable development. This because to restore and to increase the natural capital base is the best way to generate jobs, and it improves the means of survival and quality of life for all, the same for the economy.

\section{Keywords}

Ecological restoration, nature conservation, natural capital, species conservation.

\section{RÉSUMÉ}

La restauration écologique est perçue par beaucoup de conservationnistes, et par la majorité des économistes, comme une distraction, une illusion, et ce qu'est plus mauvais, comme une perte de temps et d'argent. Dans cet article on indique que la restauration, de fait, complète non seulement à la conservation de la nature mais encore elle est utile pour réussir un développement socio-économiquement durable et équitable. Cet est à cause de que restaurer et augmenter la base du capital naturel est la meilleure voie pour générer emplois, et améliorer les moyens de survivance et de qualité de vie pour tous, de la même façon pour la économie.

\section{Mots-clés}

Restauration écologique, conservation naturelle, capital naturel, conservations des espèces. 


\section{INTRODUCCIÓN}

En los países en desarrollo, donde ocurre la mayoría de estados críticos en biodiversidad tanto en la conservación de la naturaleza como en la restauración de ecosistemas degradados, será posible encontrar apoyo sólo si están claramente vinculados con el desarrollo socio-económico. Por el contrario, hablar de desarrollo socio-económico en los lugares de estos países en los que el medio ambiente está deteriorado, sin duda que exigirá una restauración ecológica de la base del capital natural. Por lo tanto, los procesos para la conservación de la naturaleza, la restauración ecológica y las políticas de desarrollo económico sostenible, se deben planear, presupuestar y ejecutar conjuntamente.

La Restauración Ecológica -RE- se puede definir como "el proceso de ayudar a la recuperación de un ecosistema que ha sido degradado, dañado 0 destruido" [1]. A esta escueta definición, es conveniente añadir que la $R E$ es un proceso que recupera y mejora la funcionalidad de los ecosistemas al interior de los paisajes que abarcan tierras en producción agrícola, así como apartar tierras para reservas naturales. La restauración de la ecología es la ciencia detrás de $-\mathrm{y}$ junto a- la práctica de RE. Ayudar a la recuperación del ecosistema aumenta la biodiversidad y sus propios servicios en lo que tiene que ver con la escala del paisaje. La mayoría de los esfuerzos de conservación se centran en reservar tierras o paisajes marinos que están claramente delimitados y considerados como relativamente "intactos", "naturales" o que poseen un importante valor de conservación La restauración ecológica se refiere tanto a estas zonas reservadas y protegidas como a las porciones utilizadas de los ambientes naturales en las que viven las personas, cultivan alimentos y extraen recursos naturales [2]. El concepto de seres humanos como miembros integrales de los ecosistemas ha sido ampliamente respaldado a nivel mundial, como lo demuestra la definición que hace la UNESCO de las reservas de la biosfera como "zonas de ecosistemas terrestres y costeros en las que se promueven soluciones para conciliar la conservación de la biodiversidad con su uso sostenible". Además, RE se describió, en un reciente "llamado a la acción" emitido por el grupo para el trabajo conjunto por la restauración ecológica de la Society for Ecological Restoration International y la IUCN Commission on Ecosystem Management -SER/IUCN-, como "un medio de conservación de la biodiversidad y sostenibilidad de los medios de subsistencia". Afortunadamente, el concepto de restauración ecológica está entrando en la cultura popular, la política pública y la educación a un ritmo muy rápido, especialmente en América del Norte, Australia, África del Sur, y también en algunas partes de Europa.

Frente a nuestro rápido crecimiento de la huella ecológica, y el creciente número de crisis ecológicas en todo el mundo, la RE tiene claramente un papel clave que desempeñar en la conservación de las especies, el capital natural, y para mejorar el bienestar humano -tanto material como culturalmente- $[3,4,5]$. En este trabajo introductorio se analizan brevemente los puntos de vista de los antagonistas de la restauración, proporcionado una réplica, y luego se explica por qué la restauración, la conservación y el desarrollo económico sostenible son mutuamente complementarios, y que los límites conceptuales y prácticos entre ellos son cada vez más borrosos. Un importante paso posterior es fomentar la ciencia y la práctica en estas tres actividades interrelacionadas, así como la gama de paisajes naturales, seminaturales, culturales, etc. -que cada vez se hace más complejas- [6]. En la conclusión se ofrece una breve descripción y una visión del camino a seguir.

\section{POR QUÉ NO RESTAURAR}

Las áreas naturales están siendo convertidas y "sacrificadas" en todas partes a un ritmo cada vez más acelerado, por una población humana en constante crecimiento, para obtener beneficios económicos a corto plazo [7]. La capacidad del umbral de desarrollo socio-económico sostenible ha sido superada en la mayoría de las naciones y regiones del mundo; y debido a que la sostenibilidad social depende en última instancia de la sostenibilidad ecológica, esta tendencia debe ser revertida.

Sin embargo, los opositores de la restauración ecológica sostienen que la restauración está invadiendo lacónicamente los presupuestos para la conservación y el desarrollo rural, y que la financiación y las políticas deberían centrarse en el desarrollo sostenible y la conservación, y no en la "costosa" restauración a largo plazo. La inversión para conservar lo que queda de la naturaleza "salvaje" aparece ahora como de sentido común, y sigue siendo de alta prioridad entre los conservacionistas [8]. Muchos ecologistas absortos, así como los intereses del desarrollo, argumentan que la restauración toma demasiado tiempo para tener un impacto significativo sobre la conservación necesaria 0 sobre los programas de desarrollo económico. Otros, particularmente los motivados por sentimientos idealistas, rechazan el pragmatismo económico y lo encuentran ofensivo al "poner un precio a la naturaleza" $[9,10]$.

Sin embargo, en los países en desarrollo -donde se concentra la mayoría de la diversidad biológica, la pobreza, la desnutrición, la guerra y las enfermedades, y donde aparece la mayoría de "puntos calientes" de la diversidad biológica- para la restauración de ecosistemas y paisajes degradados, y para la conservación de la naturaleza, solamente se encuentra apoyo si los programas están claramente vinculados con la investigación socio-económica y el desarrollo, así como con la creación de empleo y la formación. Por lo tanto, las razones holísticas y los argumentos integrados para la restauración deben articularse y comunicarse. En este trabajo se sostiene que la inversión continua de capital financiero, humano y social en la restauración de ecosistemas dañados y empobrecidos se ha convertido en esencial para 
asegurar nuestra sostenibilidad ecológica [11, 12, 13]. El desarrollo y el uso de los recursos simplemente no pueden ser sostenibles a menos que estén diseñados y gestionados de forma que se reparen los daños cada vez que ocurren. Esa es una política pragmática de supervivencia.

\section{RAZONES PRAGMÁTICAS Y ENFOQUE ESTRATÉGICO DEL POR QUÉ RESTAURAR EL CAPITAL NATURAL}

Clewell y Aronson [14] describen cinco motivaciones principales para restaurar los ecosistemas degradados: el idealista, el tecnocrático, el heurístico, el biótico y el pragmático. La base lógica pragmática para la restauración ecológica se ha descrito como un conjunto de dos componentes: la "Restauración del Capital Natural" -RCN- y el mejoramiento climático. Sin embargo, el enfoque de la RCN para la restauración ecológica puede efectivamente ser visto como un paraguas estratégico con muchos radios, incluyendo las otras motivaciones señaladas anteriormente. Cada radio representa una táctica diferente que puede y va a variar en importancia relativa en función de la escala específica, el sitio, y el contexto en cuestión. Al igual que una persona sosteniendo un paraguas en una tormenta, la sociedad puede y debe poner un paraguas RCN para ayudar a soportar la tormenta del cambio climático global. Si, las razones idealistas, tecnocráticas, heurísticas o bióticas para restaurar se invocan por separado, entonces solamente los países ricos podrán permitirse esa inversión. Los países más pobres en vía desarrollo necesitan la restauración para aumentar el capital natural, pero existen y son valoradas, como en los países ricos, las motivaciones idealistas de la RE.

\section{ESTRATEGIAS PARA LA RCN}

1. La incorporación, es decir, llevar los beneficios y motivaciones al discurso común del público en general, para que se dé por sentado

2. Crear empleos y medios de vida, y luchar para restaurar el capital social, definido como instituciones, relaciones, redes sociales, y compartir las creencias $y$ tradiciones culturales que promueven la confianza mutua

3. Mejorar los servicios y bienes mediante plazos que beneficien a las personas

4. Utilizar aquellos enfoques que son asequibles localmente, y apropiados ecológica y culturalmente, pero teniendo conocimiento de la mentalidad y la configuración específica.

5. Recuperar los sistemas auto-sostenibles que requieren poca o ninguna gestión en curso

6. Planificar con los usuarios locales para que todos los valores tangibles e intangibles de la restauración puedan ser apreciados y soportados por la acción

Todas estas estrategias no son consideradas cuando la motivación es tecnocrática, biótica, heurística o idealista. Sin embargo, la RCN es una estrategia que abarca todas las otras motivaciones para la restauración y van más allá en sus esfuerzos por restablecer un ambiente sano, sostenible, y matricial para la empeño humano. Un ejemplo pertinente es el programa Working-for-Water en Suráfrica [15, 16], una iniciativa de obras públicas en la que más de treinta mil personas fueron empleadas para erradicar plantas leñosas exóticas invasoras -especialmente acacias australianas arraigadas- que absorben y transpiran la humedad del suelo, privando a las plantas nativas, las tierras agrícolas, y a la gente, del agua que tanto necesitan. En este programa se crearon puestos de trabajo, medios de vida y un renovado capital social, mientras que el primer paso de la restauración del paisaje natural -nativo- se está llevando a cabo a través de la eliminación de especies de plantas invasoras y exóticas que usan intensivamente el agua a lo largo de los ríos y arroyos donde proliferan. De esta manera, la restauración se abre una nueva frontera para los conservacionistas y economistas por igual para encontrar una causa común y una plataforma de I + D sobre la cual el diálogo y la cooperación. Existen problemas [17], pero este programa ofrece un modelo excepcional para el estudio. Sin embargo, en este programa la restauración se lleva a cabo como una estrategia de desarrollo, que aborda simultáneamente lo que se refiere a la conservación. De esta manera, la restauración abre una nueva frontera para los conservacionistas y economistas por igual para encontrar una causa común y una plataforma de I + D sobre la cual se lleva a cabo el diálogo y la cooperación. Existen problemas [17], pero este programa ofrece un modelo excepcional para ser estudiada.

El ecologista Daniel Janzen ha hecho contribuciones duraderas no sólo a la ecología teórica, sino también a la ciencia y la práctica de la conservación de la naturaleza y la restauración ecológica. En sus escritos, ha argumentado con fuerza en favor de la utilización de la biodiversidad forestal, de tal manera que se pueda pagar por su propia conservación [12]. Esto implica que la conservación de la naturaleza, la calidad de vida, el desarrollo y la comercialización son complementarios. Como Daily y Ellison [18] lo dicen, es posible, y se debe tratar de "hacer rentable la conservación" [19]. Sin embargo, esto requerirá cambios profundos en nuestros modelos económicos y en las políticas públicas basadas en ellos [20,21].

La restauración es el reconocimiento por los seres humanos que han usado demasiado el capital natural y que -por su propio bien- ha llegado el momento para "devolver" a la naturaleza las funciones naturales de las que dependemos. Se trata de aumentar los esfuerzos -o acciones- del capital natural existente para complementar los esfuerzos de conservación por fuera de los enclaves de conservación demarcados, cuando y donde las reservas de capital natural estén peligrosamente agotadas (Fig. 1). 


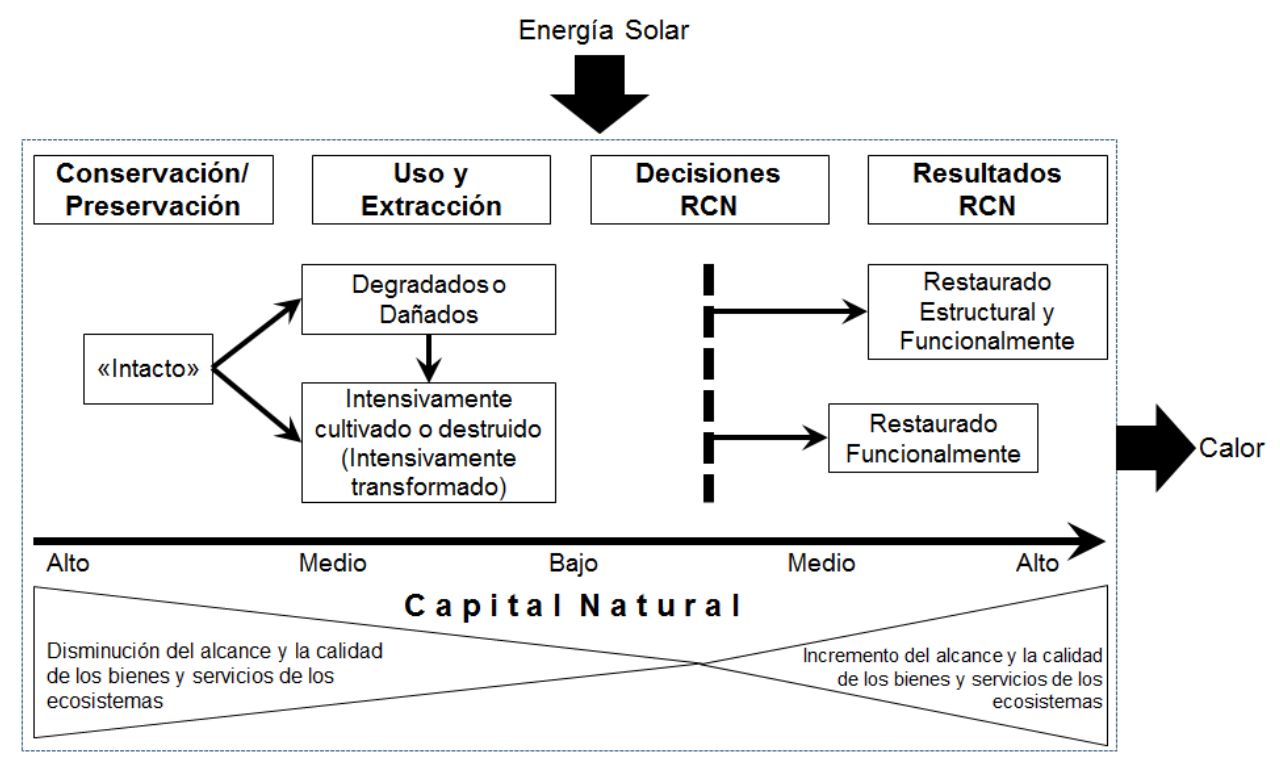

Fig. 1 Descripción esquemática del flujo de capital natural (Fuente: Autor)

Esta figura ilustra que las personas extraen y utilizan los recursos naturales en diversas formas, y a menudo lo que hacen tiene impactos negativos sobre el stock del capital natural, así como la disponibilidad de bienes y servicios del ecosistema. La dificultad y el costo de restaurar el capital natural generalmente es una función del grado de degradación [20, 22]. Esta consecuencia debe considerarse cuando se planea y presupuesta para usar o extraer recursos naturales. Las comunidades y las sociedades también pueden decidir compensar los impactos negativos de la extracción de recursos mediante la inversión en protección del ecosistema, tecnologías de uso sostenible, y la restauración del capital natural. En la parte superior izquierda de la Fig. 1, la decisión para conservar refleja el deseo de proteger razonable para proteger el capital natural remanente de los ecosistemas intactos. Más a la derecha, decisiones RCN refleja la intervención política y administrativa inspirada en las diferentes motivaciones para restaurar los ecosistemas degradados. El nivel de inversión financiera y el compromiso social, así como las limitaciones biofísicas, determinarán el grado de conservación, restauración o incremento del capital natural -bajo, medio, alto. Insistir en el enfoque de capital natural para estos problemas puede ayudar a focalizar las discusiones previas a las decisiones colectivas.

La restauración ecológica es presentada como una estrategia que aumenta el capital natural. Invertir en la restauración del capital natural no desvirtúa a la conservación de la naturaleza, sino que añade un significado adicional, la pertinencia y la eficacia de los esfuerzos de conservación. Por ejemplo, la restauración de los corredores entre las áreas protegidas desarticulada puede mantener una población que, de otro modo, podría desaparecer debido a la fragmentación del hábitat. Además, la restauración puede proporcionar nuevos puestos de trabajo, incluso a medida que aumenta el stock de bienes y servicios naturales del que todas las economías dependen. La restauración ecológica -si se holísticamente-, incrementa las oportunidades y beneficios económicos, al tiempo que mejora los aspectos sociales, culturales, psicológicos y espirituales del bienestar humano.

\section{CONCLUSIONES}

En resumen, la restauración del capital natural bien concebida y ejecutada requiere la fusión de las justificaciones tecnocráticas, heurísticas, bióticas e idealistas, así como del pragmatismo. Para ello, las instituciones con conducta de restauración tecnocrática deben renunciar a alguna autoridad y trabajar activamente en colaboración con las partes interesadas. Por el contrario, las partes interesadas particularmente los ciudadanos locales- deben estar motivados para asumir la responsabilidad social e inyectar proyectos de restauración con idealismo, significado cultural y el valor heurístico. A través de este enfoque de coalición, se cumplen las prioridades tanto de los conservacionistas, que buscan maximizar el crecimiento neto de la biodiversidad y la función del sistema, como de los economistas que buscan maximizar la rentabilidad financiera y promover el crecimiento económico como un medio para crear oportunidades de empleo, para desterrar la pobreza, el hambre y la falta de vivienda, y para mejorar la vida de la mayoría de las personas.

\section{REFERENCIAS}

[1] SER. "The SER international primer on ecological restoration". Society for Ecological Restoration, Science and Policy Working Group. 2004. Disponible en: http://www.ser.org/pdf/primer3.pdf [Nov. 2010).

[2] J. N. Blignaut, J. Aronson, S. Archer, A. F. Clewell, P. Woodworth \& N. Desai. (in press). "The values and ethics of restoring natural capital". 2007. In J. Aronson, S. J. Milton \& J. Blignaut (Eds.) Restoring Natural Capital: Science, Business and Practice. Washington: Island Press. Cap. 2.

[3] J. A. Harris, R. J. Hobbs, E. Higgs \& J. Aronson. "Ecological restoration and global climate change". Restoration Ecolog, Vol. 14, No. 2, pp. 170-176. 2006. 
[4] P. Leigh. "The ecological crisis, the human condition, and community-based restoration as an instrument for its cure". Ethics in Science and Environmental Politics 2005, pp. 3-15, Apr. 4, 2005.

[5] J. Van Andel \& J. Aronson. "Restoration ecology: The new frontier". Oxford: Blackwell Science. 2006.

[6] A. Machado. "An index of naturalness". Journal for Nature Conservation, Vol 12, No. 2, pp. 95-110. 2004.

[7] Millennium Ecosystem Assessment. "Ecosystems and human well-being: Synthesis". Washington: Island Press. 2005.

[8] A. Balmford, A. Bruner, P. Cooper, R. Costanza, S. Farber, R. E. Green. "Economic reasons for conserving wild nature". Science 9, Vol. 297, No. 5583, pp. 950-953. 2002.

[9] M. J. Stevenson. "Problems with natural capital: A response to Clewell". Restoration Ecology, Vol. 8, No. 3, pp. 211-213. 2000.

[10] J. Aronson \& E. Le Floc'h. "Restoration of natural capital: Pros and problems". Restoration Ecology, Vol. 8, No. 3, pp. 214-216. 2000.

[11] J. Cairns. "Ecological restoration: Replenishing our national and global ecological capital". In D. Saunders, R. Hobbs, \& P. Ehrlich (Eds.) Nature conservation 3: Reconstruction of fragmented ecosystems. Chipping Norton: Surrey Beatty \& Sons, pp. 193-208. 1993.

[12] D. H. Janzen. "Tropical dry forest restoration: Area de Conservacion Guanacaste, northwestern Costa Rica". In M. R. Perrow, \& A. J. Davy (Eds.) Handbook of ecological restoration. Restoration in practice. Cambridge: Cambridge University Press. Vol. 2, pp. 559-584. 2002.
[13] R. Repetto. "Government policy, economics and the forest sector". In K. Ramakrishna, \& G. Woodwell (Eds.) Forests for the future: Their use and conservation. New York: Yale University Press, pp. 93-110. 1993.

[14] A. F. Clewell \& J. Aronson. "Motivations for the restoration of ecosystems". Conservation Biology, Vol. 20, No. 2, pp. 420-428. 2006.

[15] S. J. Milton, W. R. J. Dean \& D. M. Richardson. "Economic incentives for restoring natural capital in southern African rangelands". Frontiers in Ecology and the Environment, Vol. 1, No. 5, pp. 247-254. 2003.

[16] V. W. Van Wilgen, M. P. de Wit, H. J. Anderson, D.C. Le Maitre, I. M. Kotze, S. Ndala. "Costs and benefits of biological control of invasive alien plants: Case studies from South Africa". South African Journal of Science, Vol. 100, No. 1-2, pp. 113-122. 2004.

[17] P. Woodworth. "What price ecological restoration". The Scientist, Vol. 20, No. 4, pp. 38-45.2006.

[18] G. C. Daily \& K. Ellison. "The new economy of nature". Washington: Island Press. 2002.

[19] K. Holl \& R. Howarth. "Paying for restoration". Restoration Ecology, Vol. 8, No. 3, pp. 260-267. 2000.

[20] J. Aronson, S. J. Milton \& J. N. Blignaut. "Restoring natural capital: Science, business and practice". Washington: Island Press. 2007.

[21] S. J. Milton, J. Aronson \& J. Blignaut. "Unite to save Earth's capital". Quest (South African Academy of Science), Vol. 2, No. 1, pp. 39-41. 2005.

[22] R. J. Hobbs \& D. A. Norton. "Towards a conceptual framework for restoration ecology". Restoration Ecology, Vol. 4, No. 2, pp. 93-110. 1996. 